\title{
REMOTE POWER TRANSFER IN ELECTRICAL VEHICLE BY USING SOLAR ENERGY
}

\author{
K. Purbia, D.Joshi \\ UG Students, \\ Geetanjali Institute of Technical Studies, Udaipur, Rajasthan, India \\ S. Bhadviya \\ Assistant Professor \\ Geetanjali Institute of Technical Studies, Udaipur, Rajasthan, India
}

\begin{abstract}
Remote Power Transfer [RPT] utilizing the attractive acceptance innovation which could maintain a strategic distance from human from the unsafe mishap caused because of the utilizing of links. By the utilizing of MOSFET switches in the inverter, it makes the yield with high recurrence improves the effectiveness of intensity moves between the curls. Because of this making of yield with higher recurrence the charging of battery will be quick and productive. By associating the sun oriented board to the stockpile battery, it will be charged persistently utilizing this board. This will helps the clients for the relentless driving. These advances make the RPT extremely appealing to the electric vehicle charging application in both stationary and dynamic charging. By presenting RPT in electric vehicle, the snags of charging time, range, and cost can be effectively overseen. RPT innovation is growing quickly as of late.
\end{abstract}

Key words: Static charging, Dynamic charging, Solar board, High recurrence yield by utilizing inverter, Remote Power Transfer (RPT), Safety rules.

\section{INTRODUCTION}

Presently a-day the world confronting the most difficult issue is the vitality request. Rather than this we had different procedures for the age of vitality which ought to be dangerous to our condition. So we step into the Nonsustainable power source which will be eco- accommodating to our condition. Our principle request will be the fills utilized in autos and it makes the significant effect our condition, so we present the half breed variant of Nonsustainable power source in the vehicle as electric vehicle which acts eco-accommodating to our condition.

We utilize both the blend of remote force move and the sunlight based vitality which encourages us the client for relentless driving. In an electric vehicle the battery is too difficult to even consider designing because of its high vitality thickness and force thickness. Presently a-days there are numerous kinds of batteries utilized in the instruments yet the lithium-particle batteries gives the most appropriate answer for the electric vehicles. As of late the Wireless Power Transmission has been a best point in the transportation framework. This paper begins with an essential idea of Wireless Power Transfer and it gives a short outline of Wireless Power Transfer framework and it incorporates the Magnetic enlistment standards, Existing and Proposed framework, High recurrence power yield, Solar board and some different issues like wellbeing contemplations. By presenting the most recent accomplishments in Wireless Power Transfer, we trust that this will accomplish in everywhere throughout the world.

\section{SIGNIFICANCE OF THE STUDY}

Presently a-day the term 'remote' turns into the most progressive and imaginative research field. This will assist the individuals with freeing from irritating wires and to keep them away from presenting to dangerous mishaps. which happen because of the utilizing of links. It will help the client for utilizing the electronic gadgets with no translation and confinements. It will be half breed with the sunlight based vitality and actualized in the automobiles will made the vehicle eco benevolent to the encompassing. This remote force move likewise has the advances of both stationary and dynamic charging of the batteries.

\section{SCOPE OF THE STUDY}

A worldwide temperature alteration turns into a most risky issue in now daily. This expands the warmth in the earth surface and makes the ice tops to dissolve down this builds the ocean level will be perilous to the whole world for the most part due to the contamination. The fundamental contaminating variable is the automobiles which transmit the carbon monoxide had a destructive effect on the earth. So we 
had presenting the electric vehicle with the mix of 'remote' power move made the EV high effective and it will make the contamination free condition. It additionally shields the individuals from breathing in of unsafe carbon monoxide and leads a people to live in a solid situation.

\section{BLOCK DIAGRAM}

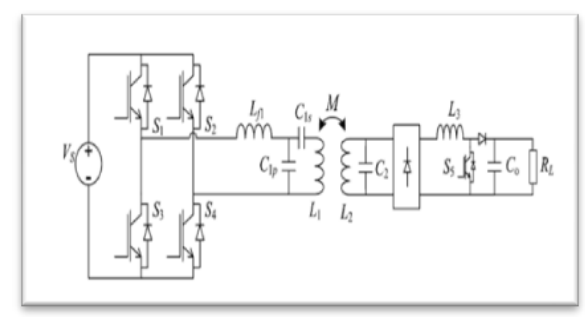

Fig. 1 Block of Wireless Power Transmission

In our project, block diagram consist of two types namely,

1. Primary side

2. Secondary side

\section{A. Primary Side}

The photovoltaic (or) Solar panel will be utilized in the introduction procedure of the circuit which produces the force for the entire activity. It ingests vitality from the sunlight based radiation and this warmth vitality will be changed over into electrical vitality, this will be provided all through the circuit. Inverters will be utilized for changing over the $\mathrm{DC}$ to $\mathrm{AC}$, power created by the sun oriented board will be the DC, these DC force will change over into AC power which it is given to the curl. The inverters comprise of four n-channel MOSFET switches these switches needs the activating heartbeats for the $\mathrm{ON}$ and OFF procedure, these activating heartbeats will be produced utilizing the PIC controller. This PIC controller will produce an activating beat of $5 \mathrm{v}$ DC. In any case, the MOSFET switches needs at least $10-12 \mathrm{v}$ DC for working the voltages. For the enhancement procedure the driver board will be utilized. Yield of the PIC controller will be given to the driver board, it begins enhancing the activating heartbeat that the yield of the driver board will be given to the inverter. This driver board yield will be associated with the door terminal of the MOSFET switches. After the change of intensity it will be moved to the curl through the pay arrange.

\section{B. Secondary Side}

The loop from the essential side gets stimulate and it makes the attractive field around the curl. Because of the utilizing of high recurrence yield, the production of attractive transition will be extremely solid. The transition from the essential curl interfaces the auxiliary loop. Thus the force will be moved between the loops through the attractive field.
Next the force from the optional loop given to the rectifier. After the rectifier the LED which demonstrate the force move to the curl. By utilizing the rectifier AC supply will be changed over into DC supply and afterward it is given to the supporter circuit answerable for the consistent yield. After that it will be shifted by utilizing the remuneration organize lastly associated with the DC shunt engine (Toy engine). The solar panel power supply will be given to the engine. Because of the persistent age of intensity by means of sun oriented board, it helps for relentless driving.

\section{CIRCUIT DIAGRAM}

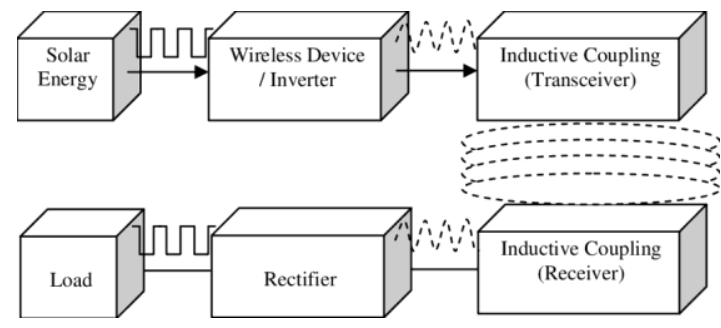

Fig. 2 Circuit of Remote Power Transfer

The photovoltaic board (PV) will be utilized as the force hotspot for the entire activity of the circuit. DC force will be created by the sun based board won't reasonable for moving in the loop. Subsequently the DC force will be given to the inverter. The inverter will comprise of MOSFET switches initiate utilizing the trigger heartbeat and convert DC to AC power. It will give the high recurrence yield to be sifted utilizing the pay system and afterward the vitality will be moved to the essential loop. Essential curl get empower which makes the motion in the loop these transition which connects the auxiliary curl and current will moved be between the loops. At that point it will be sifted and after given to the rectifier which changes over the AC capacity to DC power. Force moved will be shown by the LED and moves to the promoter circuit which gives out the consistent yield led by the diode and repaying system. At long last the ideal yield will be given to the DC engine (Toy engine).

Coupling Hypothesis: This technology is based on the working principle of mutual inductance via a two part transformer such that change in current flow through one winding induces a voltage across the ends of the other winding through electromagnetic induction.

\section{MAGNETIC COUPLER DESIGN}

Magnetic coupler for the most part utilized for move the force remotely; these are two sorts Magnetic coupler in RPT framework. One is sending side called as Primary coupler and other one is accepting side called as pick up coupler. It primarily utilized for expanding effectiveness and coupler is structured in cushion structure. The magnetic coupler relies 
upon the coupling coefficient $\mathrm{K}$ and quality factor $\mathrm{Q}$. At the point when $\mathrm{K} \& \mathrm{Q}$ esteem increments magnetic coupler effectiveness gets incremented. By expanding measurement and material high productivity can ready to accomplish. However, it isn't useful for building approach. It wanted to have higher $\mathrm{k}$ and $\mathrm{Q}$ with least measure measurement cost. Tremendous couplers were utilized in two structures. Current RPT framework utilizes in any event $10 \mathrm{kHz}$ recurrence. As the specialized advancement of intensity electronics. $100 \mathrm{kHz}$ could accomplished at high force level. At this recurrence, to lessen the air conditioner loss of copper curls wire is generally received Besides the recurrence, the coupling coefficient $\mathrm{k}$ is altogether influenced by the structure of attractive coupler. A superior coupler configuration may prompts a half $100 \%$ improvement contrasted and some no ideal plans. Coupler can be charged by two modes.

\section{Coupler in Stationary Charging}

In a stationary charging the coupler is structured in cushion structure. The early couplers are much the same as a basic split center transformer. Typically this sort of configuration could just exchange power through an extremely little hole. As per magnetic transition dispersion region the coupler could named the twofold side and single side sort. For the twofold sided type the motion goes to the two sides of coupler. At the point when the protecting is included, the quality factor of a transition pipe coupler decrease from 260 to 86. Twofold sided coupler having half of the primary motion at the back. This puts forth the protecting attempt.

Two run of the mill single side transition type cushion. One is a round cushion. Another is rectangular bipolar cushion proposed by University of Auckland; a solitary sided cushion is made out of three layers. The top layer is loop, carry the curl, a ferrite layer is embedded to upgrade and managing the motion. At the base is a protecting layer. To move power, the two cushions are put shut with loop to curl. The transition way structure of a roundabout cushion is around one-fourth of the cushion's width; DD cushion has a huge improvement in the coupling. The charge zone for a DD cushion could be around multiple times bigger than a roundabout cushion with comparable material expense. The DD cushion great tolerant in the y direction. There is invalid point for $\mathrm{DD}$ cushion in the $\mathrm{x}$ direction. A variation of a DDQ cushions which is called as new bipolar cushion. By expanding size every $\mathrm{D}$ cushion and having some cover between the two D curl, at this coupling level effectiveness above $90 \%$ could be accomplished.

The complete width of $\mathrm{W}$ type ought to be around multiple times the hole among track and pickup loop. The connection between track width and move separation is decoupled and track can be worked at a limited structure. With limited plan the development cost could be decreased. The issue of the track configuration is that pickup curl spread little segment of track, which make coupling coefficient little. The excitation of each fragment can be constrained by the switches ON-OFF state. The EMF over the idle fragments is decreased essentially. The distributed framework proficiency is about $70 \%-80 \%$, which is lower than the effectiveness accomplished in stationary charging. At the point when each section is short enough the track become like a cushion in stationary charging, which is other sort of essential attractive coupler. The essential cushion can specifically energized without a high recurrence regular current and furthermore invigorated cushion is secured by the vehicle.

\section{WIRELESS POWER TRANSFER}

Remote Power Transfer or Electromagnetic force move is the transmission of electrical vitality without utilizing the wires. Any place the interconnecting wires are badly arranged in such places Wireless force move is progressively compactable. This remote force move framework which gives the benefits of utilizing links and that could evade the short circuits, motion spillage and fire mishaps. Remote Power System comprises of different sides, transmitter and beneficiary. Basically the Resonant loops are utilized in the force move. The two loops are tuned to the equivalent resounding recurrence and the force is given to the transmitter side, full curls get stimulated and make the attractive transition or field that interfaces the loop.

\section{Coupler in Dynamic Charging}

The dynamic charging likewise called OLEVs or street way controlled electric vehicles. It has two approach to charge the EV while driving. Dynamic charging can additionally illuminates the EVs go uneasiness. In unique charging framework the attractive segment are made out of an essential side of the coupler. Which is typically covered under the street and optional side pickup loop. At the point when EV with a pickup loop is running alongside the track and it can ready to move the force proceeds. The track can be straightforward as only two wires and selection of ferrite with $\mathrm{U}$ or $\mathrm{W}$ type to build the coupling and force move distance.

\section{CONCLUSION}

In this study, we are presenting the various technologies related to Wireless Power Transfer system, which is used to avoid the flux leakage and short circuits occurred due to the cables. This will be helpful for those who are doing research in the area of wireless power transmission. The wireless Power Transmission is used to operate the cars with high efficiency and improve the quality parameters. This project is in the progress of generating power source through renewal energy. 


\section{ACKNOWLEDGEMENT}

We hereby, completed this project, would like to express our very great appreciation to Prof. S. Bhadviya for providing required guidance and his valuable and constructive suggestions during our project work. Her willingness to give his time is generously has been very much appreciated. We would also like to thank our friends for their timely help, comment and suggestions. Their suggestions and comments had really given us a new direction to work on this project.

\section{REFERENCES}

[1] Maqsood M. and Nasir N. (2013), Wireless electricity (Power) transmission using solar based power satellite technology,

Journal of Physics.

[2] Musavi F. , Eberle Wilson,(2013) Overview of wireless power transfer technologies for electric vehicle battery charging, IET Power Electronics.

[3] [3] Cheon Sanghoon, Kim Yong-Hae, Seung-Youl Kang, Lee Myung Lae, Lee Jong-Moo, and Zyung Taehyoung (7, July 2011) Circuit-Model-Based Analysis of a Wireless Energy-Transfer System via Coupled Magnetic Resonances, IEEE Transactions on Industrial Electronics, Volume: 58.

[4] [1] Tesla, N., and A. Marincic. "Nikola Tesla and the remote transmission of vitality." IEEE Trans. Force App. Syst. (101) (10) (1982): $4064-4068$.

[5] Hui, S. Y. (2013) "Planar Wireless Charging Technology for Portable Electronic Products and Qi." Proceedings IEEE (101) (6): 1290-1301.

[6] Ahn, D., and Hong S.(2014) "Remote Power Transmission with Self- Regulated Output Voltage for Biomedical Implant." IEEE Trans. Ind. Electron. (61) (5): 2225-2235.

[7] IHI Corporation. "Making Electric Vehicles Easier To Use."www.ihi.co.jp/en, (46) (1) (2013).Z. Yiming, Z. Zhengming, and C. Kainan, "Frequency decrease analysis of resonant wireless power transfer," IEEE Trans. Power Electron., Vol. 29, No. 3, pp. 1058-1063, Mar. 2014.

[8] Cheon S. et al. (2011), "Circuit-Model-Based Analysis of a Wireless Energy Transfer System via Coupled Magnetic Resonances", IEEE Trans. Ind. Electron., vol. 58, no. 7, pp. 2906-2914.

[9] Dionigi M. and Mongiardo M. (Jun. 2011), "CAD of Efficient Wireless Power Transmission systems", Microwave Symposium Digest (MTT) IEEE MTT-S Int, pp. 1-4.

[10] Ricketts D.S., Chabalko M.J. and Hillenius A.(2013), "Experimental demonstration of the equivalence of inductive and strongly coupled magnetic resonance wireless power transfer", Appl Phys Lett, vol. 102, pp. 053904.

[11] Choudhary, Singh Vikas, Pal Satendra , (2011); Wireless Power Transmission: An Innovative Idea,
International Journal of Educational Planning \& Administration. ISSN 2249-3093 Volume 1.

[12] [Huang13] Huang Wen, Zhang Biao, Chen Xing, Huang Kama, Liu Changjun, (2013): Study on an SBand Rectenna Array for Wireless Microwave Power Transmission, Progress In Electromagnetics Research, Vol. 135, 747 -758. 